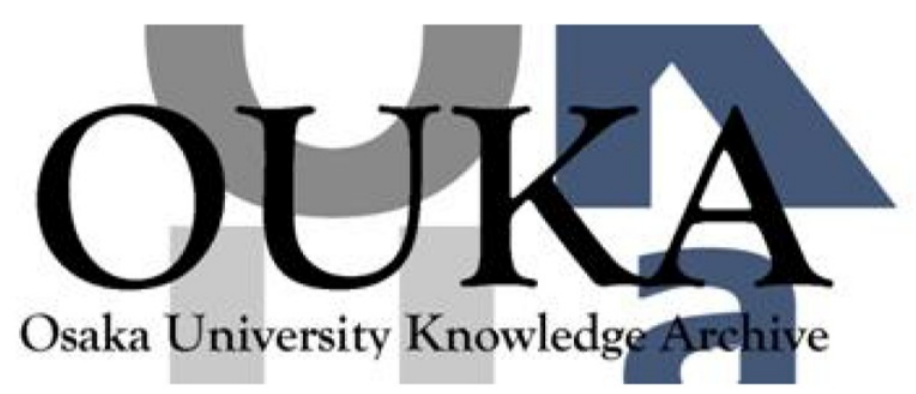

\begin{tabular}{|c|c|}
\hline Title & $\begin{array}{l}\text { Mutation spectrum and health status in skeletal } \\
\text { muscle channelopathies in Japan }\end{array}$ \\
\hline Author (s) & $\begin{array}{l}\text { Sasaki, Ryogen; Nakaza, Maki; Furuta, Mitsuru } \\
\text { et al. }\end{array}$ \\
\hline Citation & Neuromuscular Disorders. 30(7) p.546-p.553 \\
\hline Issue Date & $2020-07-01$ \\
\hline oaire:version & AM \\
\hline URL & https://hdl. handle. net/11094/85460 \\
\hline rights & $\begin{array}{l}\text { - } 2020 \text { Elsevier B.V. This manuscript version is } \\
\text { made avai lable under the Creative Commons } \\
\text { Attribution-NonCommercial-NoDerivatives } 4.0 \\
\text { International License. }\end{array}$ \\
\hline Note & \\
\hline
\end{tabular}

Osaka University Knowledge Archive : OUKA

https://ir. Library. osaka-u. ac. jp/

Osaka University 


\section{Mutation spectrum and health status in skeletal muscle channelopathies in Japan}

Ryogen Sasaki*, Maki Nakaza ${ }^{\mathrm{b}}$, Mitsuru Furuta ${ }^{\mathrm{c}}$, Haruo Fujino ${ }^{\mathrm{d}}$, Tomoya Kubota ${ }^{\mathrm{b}}$, Masanori P.

Takahashi $^{\mathrm{b} *}$

${ }^{a}$ Department of Neurology, Kuwana City Medical Center

3-11, Kotobuki-cho, Kuwana, Mie, 511-0061, Japan

${ }^{\mathrm{b}}$ Department of Functional Diagnostic Science, Osaka University Graduate School of Medicine

1-7 Yamadaoka, Suita, Osaka, 565-0871, Japan

${ }^{\mathrm{c}}$ Department of Neurology, Kansai Rosai Hospital

3-1-69. Inabasou, Amagasaki, Hyogo, 660-8511, Japan

${ }^{\mathrm{d}}$ Department of Special Needs Education, Oita University

700 Dannnoharu, Oita, Oita, 870-1192, Japan

*Corresponding authors

Ryogen Sasaki

Department of Neurology, Kuwana City Medical Center

3-11 Kotobuki-cho, Kuwana, Mie, Japan, 511-0061

Tel: +81-594-22-1211 
e-mail: rsasaki@kuwanacmc.or.jp

\author{
Masanori P. Takahashi \\ Department of Functional Diagnostic Science, Osaka University Graduate School of \\ Medicine \\ 1-7 Yamadaoka, Suita, Osaka, Japan, 5650871 \\ Tel: +81-6-6879-2587 Fax: +81-6-6879-2587 \\ e-mail: mtakahas@sahs.med.osaka-u.ac.jp
}

Declarations of interest:

MPT reports grants from Astellas, FP Pharma and Pfizer during the conduct of the study; grants from Novartis and Japan Blood Products Organization, personal fees from Alexion and Sanofi, outside the submitted work. The sponsors had no control over the interpretation, writing, or publication of this work. Other authors have nothing to disclose. 


\section{Abstract}

Skeletal muscle channelopathies, including non-dystrophic myotonia and periodic paralysis, are rare hereditary disorders caused by mutations of various ion channel genes. To define the frequency of associated mutations of skeletal muscle channelopathies in Japan, clinical and genetic data of two academic institutions, which provides genetic analysis service, were reviewed. Of 105 unrelated pedigrees genetically confirmed, 66 pedigrees were non-dystrophic myotonias [CLCN1 (n $=30)$ and $S C N 4 A(\mathrm{n}=36)], 11$ were hyperkalemic periodic paralysis $(S C N 4 A)$, and 28 were hypokalemic periodic paralysis [CACNAIS $(\mathrm{n}=16)]$ and $S C N 4 A(\mathrm{n}=12)]$. Of the 30 families with myotonia congenita, dominant form (Thomsen type) consisted $67 \%$, and unique mutations, A298T, P480T, T539A, and M560T, not found in Western countries, were commonly identified in CLCN1. Hypokalemic periodic paralysis caused by SCN4A mutations consisted $43 \%$ in Japan, which was much higher than previous reports. Furthermore, the quality of life of the patients was assessed using the patient-reported outcome measures, SF-36 and INQoL, for 41 patients. This study indicated that the etiology of skeletal muscle channelopathies in Japan was not identical to previous reports from Western countries, and provided crucial information for genetics as well as future therapeutic interventions.

\section{Keywords}


Skeletal muscle channelopathies; Non-dystrophic myotonia; Periodic paralysis; CLCN1; SCN4A;

CACNAIS

\section{Footnotes}

NDM, non-dystrophic myotonia; PP, periodic paralysis; MC, myotonia congenita; PMC,

paramyotonia congenita: SCM, sodium channel myotonia; hyperPP, hyperkalemic periodic paralysis;

hypoPP, hypokalemic periodic paralysis; 


\section{Introduction}

Skeletal muscle channelopathies are rare inherited neuromuscular disorders that are associated with mutations in the genes encoding voltage-gated ion channels expressed in skeletal muscle[1]. They are divided into two main categories according to their major clinical symptoms: nondystrophic myotonia (NDM) and periodic paralysis (PP)[2,3]. NDM includes three types of clinical disorders: myotonia congenita (MC), paramyotonia congenita (PMC), and sodium channel myotonia (SCM). MC is caused by mutations in the skeletal muscle chloride channel (CLCN1) gene, whereas PMC and SCM are caused by mutations in the skeletal muscle sodium channel (SCN4A) gene. PP comprises two types of clinical disorders that are characterized by the serum potassium concentrations during paralytic attacks: hyperkalemic periodic paralysis (HyperPP) and hypokalemic periodic paralysis (HypoPP). HyperPP is caused by the SCN4A mutations, whereas HypoPP is caused by mutations in two different genes; i.e., the SCN4A (HypoPP2) or muscle calcium channel $(C A C N A 1 S)$ gene (HypoPP1). In other words, PMC, SCM, HyperPP, and hypoPP2 are allelic disorders caused by mutations in the identical gene, SCN4A.

Many mutations in the SCN4A, CLCN1, and CACNA1S genes have been identified in patients with skeletal muscle channelopathies worldwide, but genetic etiology studies have not been often performed in Asian countries[4,5]. Additionally, several nationwide studies of skeletal muscle channelopathies in Western countries have assessed the quality of life (QOL) using patient-reported outcome (PRO) measures including the Short Form 36 Item Health Survey (SF-36) and 
Individualized Neuromuscular Quality of Life (INQoL)[6-8]. These PRO instruments were utilized in a double-blind clinical trial for skeletal muscle channelopathies as secondary endpoints and provided evidence for intervention, the beneficial effects of mexiletine[9].

To date, no systematic study has been carried out in Japan. Several case reports from Japan have suggested that the number of skeletal muscle channelopathies observed in Japan seems to be fewer than observed in Western countries, and the mutations found in Japanese patients are unique. In addition, the QOL of the patients has never been investigated. In our study, we aimed to clarify the frequency and distribution of the associated mutations in the Japanese population. We reviewed the clinical and genetic data that were collected from 1996 to 2016 by two Japanese laboratories. These laboratories have been conducting genetic analysis on skeletal muscle channelopathies at the request of medical institutions from all over Japan. Additionally, to explore the influence of the symptoms on the patient's QOL, we conducted questionnaire surveys using two types of PRO instruments that were validated in the Japanese language; i.e., the SF-36 and INQoL.

\section{Materials and Methods}

2.1. Family materials and genetic analysis

We analyzed consecutive pedigrees with a diagnosis of skeletal muscle channelopathies that was genetically confirmed between April 1996 and December 2016. We performed genetic analyses of the Japanese patients who were clinically diagnosed with NDM or PP and who were referred to our 
laboratories by medical institutions in Japan. In brief, we conducted Sanger DNA sequencing of all exons of the CLCN1 (NM_000083.3) and/or SCN4A (NM_000334.4) genes for patients with NDM and hyperPP, and of "hot spot" exons including exons 4, 11, 21 and 30 of CACNAIS

(NM_000069.3) and exons 5, 12, 13, 18, 20, 21, 23 and 24 of SCN4A for patients with hypoPP.

When a novel mutation was identified, extensive database exploration using ExAC[10] and segregation analysis was performed to confirm the pathogenicity based on ACMG guidelines[11].

For some cases, functional analyses of the mutated channel were performed, as reported previously.

To attribute the clinical phenotype; that is, either SCM or PMC, to a mutation was sometimes challenging because we observed patients with the same mutation exhibiting both phenotypes. Therefore, we considered PMC and SCM as one class of NDM, which was associated with the SCN4A mutation.

It should be noted that, in Japan, most of the genetic analysis for the patients with AndersenTawil syndrome was performed by other institutions that specialized in cardiology. This study was approved by the Ethics Committees of Mie University Hospital and Osaka University. Written informed consent was obtained from all participants before enrolling in the study.

2.3. The questionnaire survey 
Forty-one Japanese patients with clinically and genetically defined skeletal muscle

channelopathy participated in the study, comprising $18 \mathrm{MC}$ cases, 11 hypoPPs, nine PMC/SCMs and three HyperPPs.

\subsection{PRO measurements and ADL}

The QOL of the patients were assessed with the SF-36[12], and INQoL [13,14], and the ADL of those were evaluated with Barthel's index[15]

The SF-36 is a widely used generic questionnaire that assesses a patients' self-reported health status across mental, physical, and social domains[16]. There are 36 items in the questionnaire that assess eight domains (physical functioning, role limitations due to physical, body pain, general health perception, vitality, social functioning, role limitations due to emotional, mental health). In addition, there is a physical component summary score and a mental component summary score. A higher score indicates better health; that is, there is a higher level of functioning or less pain.

INQoL has been validated for adults with various neuromuscular diseases [13]. It consists of 54 items covering 13 domains; that is, seven that assess muscle symptoms (weakness, fatigue, pain, myotonia, blepharoptosis, diplopia, and dysphagia), five that evaluate the impact of the muscle disease on areas of life (activities, independence, relationships, emotions, and body image) and one that assesses treatment effectiveness. The INQoL summary score is a composite of the five domains 
that are used to assess the impact of disease on QOL. They can be thought of as a percent of the overall detrimental impact on a patient's life. A higher score indicates a worse perception of QOL.

\subsection{Statistical analysis}

Standard statistical methods were used for all descriptive statistics. These included the calculation of the median and the first and third quartiles. Subscale scores from the INQoL and SF36 were compared with the median of Kruskal-Wallis test. Correlations between the INQoL index and INQoL section1 subscale scores were investigated with Spearman correlation coefficients. All the statistical analyses were performed with JMP (SAS Institute Inc.).

\section{Results}

We identified 105 probands with $C L C N 1, S C N 4 A$, and CACNAIS mutations in unrelated pedigrees, of which $82(78 \%)$ had a positive family history. Sixty-six probands (63\%) were diagnosed with NDM (MC and PMC/SCM), whereas 39 probands (37\%) were diagnosed with PP (hyperPP, hypoPP1 and hypoPP2; Figure 1). Within NDM, 30 probands (45\%) were diagnosed with MC and 36 probands (55\%) were diagnosed with PMC/SCM. One proband with NDM had both CLCN1 and SCN4A mutations. Within PP, 28 PP probands (72\%) were diagnosed with hypoPP and 11 PP probands (28\%) were diagnosed with hyperPP. Twenty-eight hypoPP probands included 16 
(57\%) probands with CACNA1S mutations (hypoPP1) and 12 (43\%) with SCN4A mutations

(HypoPP2).

\subsection{CLCN1 mutations}

Nineteen different $C L C N 1$ mutations were detected in 30 probands, encompassing 14

missense, three nonsense, and two frameshift mutations (Table 1). Twenty-one probands were heterozygous, and three probands were homozygous, all resulting from consanguineous marriages.

The remaining six probands were compound heterozygous. It should be noted that compound heterozygous mutations (V321M/L844P, P480T/R626X, and T539A/M560T) with autosomal dominant inheritance were found in three families. As exemplified in our previous case report[17], these probands had more severe disease than their affected parent who has only one mutation. The four common variants (A298T, P480T, T539A, and M560T) accounted for 63\% of the mutations. The A298T mutation caused either a dominant or recessive pattern of inheritance. Among the 21 probands with the heterozygous mutation, five patients (R105C, P282L, and V286Gfx60, and one each of A298T and T539A; 17\%) were sporadic. R317X mutation was identified in the siblings born from apparently unaffected parents

\subsection{SCN4A mutations}


Fifty-nine probands with SCN4A mutations were classified as PMC/SCM (n=36), hyperPP $(\mathrm{n}=11)$ and hypoPP2 $(\mathrm{n}=12)$. Twenty-eight different $S C N 4 A$ mutations were detected in 59 probands with PMC/SCM ( $\mathrm{P}=21)$, hyperPP $(\mathrm{n}=3)$ and hypoPP2 $(\mathrm{n}=4)$. We identified 26 missense mutations, one intron, and one deletion mutation (Table 1). The most frequently recognized mutations were T1313M and substitutions at position 1448, including R1448C, R1448G, R1448H, and R1448P mutations, accounting for 39\% in the PMC/SCM group. T704M and M1592V mutations accounted for $91 \%$ in the hyperPP group, whereas $\mathrm{R} 669 \mathrm{H}$ and $\mathrm{R} 672 \mathrm{H}$ mutations accounted for $75 \%$ in the hypoPP2 group. Fifteen patients (25\%) were sporadic.

\subsection{CACNAIS mutations}

In 16 probands of hypoPP1 pedigrees, we identified R528H, R1239H, and R900G mutations in the S4 segments that contribute to voltage sensing (Table 1). R528H and R1239H mutations accounted for $94 \%$ of the CACNAIS mutations in the hypoPP1 group. Two patients (13\%) were sporadic.

In summary, as for PP, 39 probands had a diagnosis of 28 HypoPP and 11 HyperPP. In probands with HypoPP, a total of three CACNAIS and 4 SCN4A mutations were detected. Of these mutations, four accounted for $86 \%$ of the pedigrees. Three different $S C N 4 A$ mutations were found in probands of the HyperPP pedigrees, of which two accounted for $91 \%$ of the pedigrees. 


\subsection{PRO measurements}

Forty-one patients whose diagnoses were confirmed by the genetic analysis participated in the PRO measurements. We divided the participants into three groups based on their main clinical features and the causative gene. There are 18 in the MC group, 11 in the HypoPP group and 12 in the myotonic disorders due to an SCN4A mutation, including HyperPP (Nav-Myt; Table 2). A majority of participants were male in all of the groups.

First, the QoL index score in the INQoL did not show any significant differences among the three groups, indicating that the overall QOL measured by INQoL was not different among the three groups. The comparison of the symptom subscales in INQoL among the three groups did not show a significant difference except for the domain "myotonia" ( $\mathrm{P}=0.0099$; Table 3). Second, we determined which symptom had a significant influence on the QOL score in each group according to Spearman correlation coefficients. As a result, it was revealed that "muscle weakness" and "fatigue" had an association with the QoL index score in the HypoPP group, "fatigue" and "myotonia" in the MC group, and "muscle weakness" in the Nav-Myt group (Table 4). All scores in the SF-36 domains were not statistically different among the three groups (Table 5, Supplemental Table 2).

\section{Discussion}

Our study included one of the largest cohorts of patients with genetically defined muscle channelopathies in Japan. The frequency and spectrum of genetic alterations observed in patients 
with NDM and PP in Japan were different from those previously reported in the literature. Of 105 cases in our study, $23(22 \%)$ were sporadic. This showed that sporadic cases of muscle channelopathies are not rare.

MC is the most common skeletal muscle channelopathy[2]. There are both an autosomal dominant (Thomsen type) and autosomal recessive (Becker type) variants of this disease, with a more severe phenotype in the latter classification[18,19]. Clinical heterogeneity within a family is observed quite often. The same mutation in $C L C N 1$ has been associated with either a dominant or a recessive inheritance pattern in different pedigrees. The dominant looking or pseudo-dominant pedigrees have been explained with incomplete penetrance and, more recently, with an unrecognized additional mutation[20].

From this study, several unique aspects of MC in Japan emerged. First, most of the CLCNI mutations in Western countries are recessive and, in the majority of cases, occur as compound heterozygous mutations. For example, the majority of patients with $\mathrm{MC}$ in the Northern Scandinavian and Finnish study were compound heterozygous, even when they were in families with a dominant pattern of inheritance[21,22]. In our cohort, MC with dominant inheritance is far more than that with a recessive pattern. Three heterozygous mutations in our list, R105C, P282L, and V286GfsX60, however, could be recessive since they were identified in only sporadic cases. A298T mutation was identified in either heterozygous or compound heterozygous. Although a functional study of the A298T channel did not reveal a dominant-negative effect, two families in China also 
exhibited dominant inheritance (Supplemental Table 1.)[23] One of the limitations of our study is the lack of RNA-transcript or protein analysis. Like W118G was reclassified as moderately pathogenic[20], detailed analysis, including immunohistochemical or molecular detection of CLCN1 protein, as well as the accumulation of more pedigrees, might be necessary to fully define the mode of inheritance.

Second, F413C and R894X are the most common CLCN1 mutations identified in patients with MC in European countries[24]. However, these mutations were not detected in our Japanese cohort. In contrast, A298T, P480T, T539A, and M560T, which were the most common mutations in our cohort, were not observed in European countries. These mutations were also reported from Korea and China[4,5,23,25-28]. Thus, it is suggested that these CLCN1 mutations might preferentially exist in East Asia, including Japan.

Finally, three probands were compound heterozygous for CLCN1 mutations in families with an apparent dominant transmission. The probands in these three pedigrees had more severe phenotypes than the other affected family members. When severity in the same family is highly variable, the probands may have two mutations.

Although MC is more common than PMC/SCM, as confirmed by a study on the prevalence of skeletal muscle channelopathies in the UK, the Netherlands, Italy, and Canada[29-32], the frequency of NDM with SCN4A mutations was higher than that with CLCN1 mutations in Japan (Table 6). As mentioned above, we found a higher percentage of dominant MC pedigrees in Japan. It is likely that 
patients with mild Thomsen type disease do not visit physicians. In the three pedigrees with compound heterozygous mutations that were inherited in an autosomal manner, the non-proband patients did not visit the hospital. Mild dominant MC is more frequent than recessive MC in Japan, and so MC may have a lower incidence than PMC/SCM.

Sodium channelopathies include PMC, SCM, HyperPP, and HypoPP2, which are allelic disorders caused by SCN4A mutations. The frequency and spectrum of genetic alterations observed in patients with $S C N 4 A$ mutations were similar to those observed in other countries. Although most SCN4A mutations are missense mutations, we identified an intron and deletion mutations in the SCN4A gene in sporadic cases, which may have been de novo mutations[33]. Of note, an unusual deletion mutation in SCN4A, K880del has been identified in a sporadic case of hyperPP. This mutation could also be unique in East Asian since the same mutation was identified in a PMC family from China [28]and in an SCM patient from Japan[34].

The patient with E950K mutation in CLCN1 and F1290L in SCN4A showed NDM with PP. The affected mother of the proband did not have PP and carried the E950K mutation only[35]. The coexistence of heterozygous $C L C N 1$ and $S C N 4 A$ mutations has been described previously in two reports[35,36]. For this reason, the analysis of both genes should be considered for patients with NMD with atypical clinical and neurophysiological features.

HypoPP is the most common type of PP. Inheritance is autosomal dominant, with reduced penetrance in females. The spectrum of mutations in patients with hypoPP1 and hypoPP2 was 
similar to those reportedpreviously[37], but the frequency of hypoPP1 and hypoPP2 in Japan was different from that reported in European countries (Table 6)[37-39]. In hypoPP1, more than six mutations of the CACNAIS gene have been described, and they account for the majority of cases. In hypoPP2, more than eight mutations of the SCN4A gene have been described, accounting for $10 \%$ $25 \%$ of cases. In our study, the frequency of hypoPP 2 was $43 \%$. This study indicates that the SCN $4 A$ mutations in patients with hypoPP in Japan are more frequent than in European countries.

Regarding PRO measurements, no statistical difference was detected among the three groups, MC, HypoPP, and Nav-Myt composed of PMC/SCM and HyperPP. The reason why is most likely due to the limited number of cases in this study compared with previous studies conducted in Western countries. The comparison of subscales of INQoL between this study and the previous study in the U.S. revealed that domains associated with "symptoms" including pain, fatigue, and myotonia in this study were milder than those in the previous study[6-8]. On the other hand, domains related to 'daily life' including activities, independence, relationship, emotions, and body image were similar between the two studies. This indicates that recognition of the symptoms vary, and it is possible that even patients who recognize the milder symptoms did not have a good outcome in daily life. The QOL in the Japanese patents with skeletal muscle channelopathy might relate more to the social issues compared to patients in the US. The critical factor to improve QOL in skeletal muscle channelopathy will be explored using several approaches $[9,40]$. Further research is required to assess and improve QOL in skeletal muscle channelopathy. One of the feasible approaches is PRO 
measurements using a real-time input system employing the internet, which would not be paperbased[41].

\section{Conclusions}

In our cohort, the mutation spectrum in skeletal muscle channelopathies in Japan was different from that in Western countries. In NDM dominant pedigrees, MC was more frequent than recessive MC and unique mutations were found in Japan. In PP, hypoPP2 was more frequent than hypoPP1.

\section{Acknowledgments}

All authors thank Makoto Ikejiri, Maki Nakamura, Kimie Hayashi, and Terumi Kikuchi for their technical supports and Dr. Kaname Nakatani for his invaluable suggestions and help.

\section{Funding}

This work was supported by Research Grants from MHLW (H29-Nanchitou(Nan)-Ippan-030), the AMED (JP19ek0109230) and JSPS KAKENHI 15K09314 (M.P.T).

\section{Author contributions}


R.S., M.N., M.F., T.K., and H.F. performed the data analysis. M.N. and H.F. conducted statistical analysis. R.S. and M.P.T. designed the study plan. R.S., M.N., M.F., T.K., H.F., and M.P.T. interpreted data. R.S., M.N., and T.K. wrote the manuscript with help of H.F. and M.P.T. 


\section{References}

[1] Cannon SC. Channelopathies of skeletal muscle excitability. Compr Physiol 2015;5:761-90. doi:10.1002/cphy.c140062.

[2] Matthews E, Fialho D, Tan S V, Venance SL, Cannon SC, Sternberg D, et al. The nondystrophic myotonias: molecular pathogenesis, diagnosis and treatment. Brain 2010;133:9-22. doi:10.1093/brain/awp294.

[3] Venance SL, Cannon SC, Fialho D, Fontaine B, Hanna MG, Ptacek LJ, et al. The primary periodic paralyses: diagnosis, pathogenesis and treatment. Brain 2006;129:8-17. doi:10.1093/brain/awh639.

[4] Nam T-S, Jung H-J, Choi S-Y, Kim Y-O, Kim M-K, Cho K-H. Clinical Characteristics and Analysis of CLCN1 in Patients with \&quot;EMG Disease\&quot;. J Clin Neurol 2012;8:212-7. doi:10.3988/jen.2012.8.3.212.

[5] Liu X-L, Huang X-J, Shen J-Y, Zhou H-Y, Luan X-H, Wang T, et al. Myotonia congenita: novel mutations in CLCN1 gene. Channels (Austin) 2015;9:292-8. doi:10.1080/19336950.2015.1075676.

[6] Sansone VA, Panzeri M, Montanari M, Apolone G, Gandossini S, Rose MR, et al. Italian validation of INQoL, a quality of life questionnaire for adults with muscle diseases. Eur J Neurol 2010;17:1178-87. doi:10.1111/j.1468-1331.2010.02992.x. 
[7] Sansone VA, Ricci C, Montanari M, Apolone G, Rose M, Meola G, et al. Measuring quality of life impairment in skeletal muscle channelopathies. Eur J Neurol 2012;19:1470-6. doi:10.1111/j.1468-1331.2012.03751.x.

[8] Trivedi JR, Bundy B, Statland J, Salajegheh M, Rayan DR, Venance SL, et al. Non-dystrophic myotonia: prospective study of objective and patient reported outcomes. Brain 2013;136:2189-200. doi:10.1093/brain/awt133.

[9] Statland JM, Bundy BN, Wang Y, Rayan DR, Trivedi JR, Sansone VA, et al. Mexiletine for symptoms and signs of myotonia in nondystrophic myotonia: a randomized controlled trial. JAMA 2012;308:1357-65. doi:10.1001/jama.2012.12607.

[10] Exome Aggregation Consortium n.d. http://exac.broadinstitute.org/.

[11] American College of Medical Genetics and Genomics n.d. https://www.acmg.net/ACMG/Medical-Genetics-Practice-Resources/PracticeGuidelines.aspx.

[12] Fukuhara S, Bito S, Green J, Hsiao A, Kurokawa K. Translation, adaptation, and validation of the SF-36 Health Survey for use in Japan. J Clin Epidemiol 1998;51:1037-44.

[13] Vincent KA, Carr AJ, Walburn J, Scott DL, Rose MR. Construction and validation of a quality of life questionnaire for neuromuscular disease (INQoL). Neurology 2007;68:1051-7. doi:10.1212/01.wnl.0000257819.47628.41. 
[14] Fujino H, Saito T, Takahashi MP, Takada H, Nakayama T, Ogata K, et al. Validation of The Individualized Neuromuscular Quality of Life in Japanese patients with myotonic dystrophy. Muscle Nerve 2018;58:56-63. doi:10.1002/mus.26071.

[15] Mahoney FI, Barthel DW. Functional evaluation: The Barthel Index. Md State Med J $1965 ; 14: 61-5$.

[16] Ware JE, Sherbourne CD. The MOS 36-item short-form health survey (SF-36). I. Conceptual framework and item selection. Med Care 1992;30:473-83.

[17] Sasaki R, Takahashi MP, Kokunai Y, Hirayama M, Ibi T, Tomimoto H, et al. Compound heterozygous mutations in the muscle chloride channel gene (CLCN1) in a Japanese family with Thomsen's disease. Clin Neurol 2013;53:316-9. doi:10.5692/clinicalneurol.53.316.

[18] Zhang J, George AL, Griggs RC, Fouad GT, Roberts J, Kwieciński H, et al. Mutations in the human skeletal muscle chloride channel gene (CLCN1) associated with dominant and recessive myotonia congenita. Neurology 1996;47:993-8. doi:10.1212/wnl.47.4.993.

[19] Plassart-Schiess E, Gervais A, Eymard B, Lagueny A, Pouget J, Warter JM, et al. Novel muscle chloride channel (CLCN1) mutations in myotonia congenita with various modes of inheritance including incomplete dominance and penetrance. Neurology 1998;50:1176-9. doi:10.1212/wnl.50.4.1176. 
[20] Raheem O, Penttilä S, Suominen T, Kaakinen M, Burge J, Haworth A, et al. New immunohistochemical method for improved myotonia and chloride channel mutation diagnostics. Neurology 2012;79:2194-200. doi:10.1212/WNL.0b013e31827595e2.

[21] Sun C, Tranebjaerg L, Torbergsen T, Holmgren G, Van Ghelue M. Spectrum of CLCN1 mutations in patients with myotonia congenita in Northern Scandinavia. Eur J Hum Genet 2001;9:903-9. doi:10.1038/sj.ejhg.5200736.

[22] Papponen H, Toppinen T, Baumann P, Myllylä V, Leisti J, Kuivaniemi H, et al. Founder mutations and the high prevalence of myotonia congenita in northern Finland. Neurology 1999;53:297-302. doi:10.1212/wnl.53.2.297.

[23] Gao F, Ma FC, Yuan ZF, Yang CW, Li HF, Xia ZZ, et al. Novel chloride channel gene mutations in two unrelated Chinese families with myotonia congenita. Neurol India 2010;58:743-6. doi:10.4103/0028-3886.72163.

[24] Meyer-Kleine C, Steinmeyer K, Ricker K, Jentsch TJ, Koch MC. Spectrum of mutations in the major human skeletal muscle chloride channel gene (CLCN1) leading to myotonia. Am J Hum Genet 1995;57:1325-34.

[25] Sasaki R, Ito N, Shimamura M, Murakami T, Kuzuhara S, Uchino M, et al. A novel CLCN1 mutation: P480T in a Japanese family with Thomsen's myotonia congenita. Muscle Nerve 2001;24:357-63. 
[26] Tsujino A, Kaibara M, Hayashi H, Eguchi H, Nakayama S, Sato K, et al. A CLCN1 mutation in dominant myotonia congenita impairs the increment of chloride conductance during repetitive depolarization. Neurosci Lett 2011;494:155-60. doi:10.1016/j.neulet.2011.03.002.

[27] Chin HJ, Kim CH, Ha K, Shin JH, Kim D-S, So I. Electrophysiological characteristics of R47W and A298T mutations in CLC-1 of myotonia congenita patients and evaluation of clinical features. Korean J Physiol Pharmacol 2017;21:439-47. doi:10.4196/kjpp.2017.21.4.439.

[28] Yang X, Jia H, An R, Xi J, Xu Y. Sequence CLCN1 and SCN4A in patients with Nondystrophic myotonias in Chinese populations: Genetic and pedigree analysis of 10 families and review of the literature. Channels 2017;11:55-65. doi:10.1080/19336950.2016.1212140.

[29] Dupré N, Chrestian N, Bouchard J-P, Rossignol E, Brunet D, Sternberg D, et al. Clinical, electrophysiologic, and genetic study of non-dystrophic myotonia in French-Canadians. Neuromuscul Disord 2009;19:330-4. doi:10.1016/j.nmd.2008.01.007.

[30] Stunnenberg BC, Raaphorst J, Deenen JCW, Links TP, Wilde AA, Verbove DJ, et al. Prevalence and mutation spectrum of skeletal muscle channelopathies in the Netherlands. Neuromuscul Disord 2018;28:402-7. doi:10.1016/j.nmd.2018.03.006. 
[31] Horga A, Raja Rayan DL, Matthews E, Sud R, Fialho D, Durran SCM, et al. Prevalence study of genetically defined skeletal muscle channelopathies in England. Neurology 2013;80:14725. doi:10.1212/WNL.0b013e31828cf8d0.

[32] Maggi L, Lo Monaco M, Portaro S, Meola G, Desaphy J, Lucchiari S, et al. Prevalence study of muscle channelopathies in Italy. Neuromuscul Disord 2016;26:S197. doi:10.1016/j.nmd.2016.06.400.

[33] Kubota T, Roca X, Kimura T, Kokunai Y, Nishino I, Sakoda S, et al. A mutation in a rare type of intron in a sodium-channel gene results in aberrant splicing and causes myotonia. Hum Mutat 2011;32:773-782. doi:10.1002/humu.21501.

[34] Kibe T, Ohtaka K, Misawa C, Iketani M, Ito Y, Mori Y, et al. A Case of myotonia permanens with a variant in SCN4A gene. No to Hattatsu, 2010; 42:388 (Conference Pap. Japanese only).

[35] Kato H, Kokunai Y, Dalle C, Kubota T, Madokoro Y, Yuasa H, et al. A case of nondystrophic myotonia with concomitant mutations in the SCN4A and CLCN1 genes. J Neurol Sci 2016;369:254-8. doi:10.1016/j.jns.2016.08.030.

[36] Furby A, Vicart S, Camdessanché JP, Fournier E, Chabrier S, Lagrue E, et al. Heterozygous CLCN1 mutations can modulate phenotype in sodium channel myotonia. Neuromuscul Disord 2014;24:953-9. doi:10.1016/j.nmd.2014.06.439. 
[37] Matthews E, Labrum R, Sweeney MG, Sud R, Haworth A, Chinnery PF, et al. Voltage sensor charge loss accounts for most cases of hypokalemic periodic paralysis. Neurology 2009;72:1544-7. doi:10.1212/01.wnl.0000342387.65477.46.

[38] Davies NP, Eunson LH, Samuel M, Hanna MG. Sodium channel gene mutations in hypokalemic periodic paralysis: an uncommon cause in the UK. Neurology 2001;57:1323-5. doi:10.1212/wnl.57.7.1323.

[39] Sternberg D, Maisonobe T, Jurkat-Rott K, Nicole S, Launay E, Chauveau D, et al. Hypokalaemic periodic paralysis type 2 caused by mutations at codon 672 in the muscle sodium channel gene SCN4A. Brain 2001;124:1091-9. doi:10.1093/brain/124.6.1091.

[40] Burns TM, Graham CD, Rose MR, Simmons Z. Quality of life and measures of quality of life in patients with neuromuscular disorders. Muscle Nerve 2012;46:9-25. doi:10.1002/mus.23245.

[41] Coathup V, Teare HJA, Minari J, Yoshizawa G, Kaye J, Takahashi MP, et al. Using digital technologies to engage with medical research: views of myotonic dystrophy patients in Japan. BMC Med Ethics 2016;17:51. doi:10.1186/s12910-016-0132-2. 
Figure legend

Fig.1 Frequency distributions in the three channelopathy categories and sodium channelopathy subtypes in Japan. 
Table 1. Frequency of variants in the probands with muscle channelopathies in Japan

\begin{tabular}{|c|c|c|c|c|c|c|c|c|c|c|c|c|c|}
\hline \multicolumn{5}{|c|}{$\begin{array}{c}C L C N 1 \\
\mathrm{n}=30\end{array}$} & & \multicolumn{6}{|c|}{$\begin{array}{c}S C N 4 A \\
\mathrm{n}=59\end{array}$} & \multicolumn{2}{|c|}{$\begin{array}{c}C A C N A 1 S \\
\mathrm{n}=16\end{array}$} \\
\hline $\begin{array}{l}\text { heterozygous } \\
\qquad \mathrm{n}=21\end{array}$ & $\mathrm{n}$ & $\begin{array}{c}\text { homozygous } \\
\mathrm{n}=3\end{array}$ & $\mathrm{n}$ & $\begin{array}{l}\text { compound hetero. } \\
\qquad \mathrm{n}=6\end{array}$ & $\mathrm{n}$ & $\begin{array}{l}\mathrm{PMC} / \\
\mathrm{SCM} \\
\mathrm{n}=36\end{array}$ & $\mathrm{n}$ & $\begin{array}{c}\text { hyperPP } \\
\mathrm{n}=11\end{array}$ & $\mathrm{n}$ & $\begin{array}{c}\text { hypoPP2 } \\
\mathrm{n}=12\end{array}$ & $\mathrm{n}$ & $\begin{array}{c}\text { hypoPP1 } \\
\text { n=16 }\end{array}$ & $\mathrm{n}$ \\
\hline $\mathrm{R} 105 \mathrm{C} \quad$ (nc) & 1 & Q445X & 1 & $\mathrm{R} 47 \mathrm{~W} / \mathrm{A} 298 \mathrm{~T}$ & 1 & V445M & 2 & $\mathrm{~T} 704 \mathrm{M}$ & 4 & $\mathrm{R} 669 \mathrm{H}$ & 4 & $\mathrm{R} 528 \mathrm{H}$ & 11 \\
\hline P282L (nc) & 1 & R626X & 1 & $\mathrm{C} 254 \mathrm{~W} / \mathrm{A} 298 \mathrm{~T}$ & 1 & A699T & 1 & K880del & 1 & $\mathrm{R} 672 \mathrm{H}$ & 5 & R900G & 1 \\
\hline V286GfsX60(nc) & 1 & V851M & 1 & E291K/R453PfsX14 & 1 & V1149L & 1 & M1592V & 6 & R1132Q & 1 & $\mathrm{R} 1239 \mathrm{H}$ & 4 \\
\hline A298T & 2 & & & V321M/L844P (AD) & 1 & $\mathrm{I} 1157 \mathrm{~N}$ & 1 & & & R1135H & 2 & & \\
\hline R317X & 1 & & & $\mathbf{P 4 8 0 T / R 6 2 6 X ~ ( A D ) ~}$ & 1 & F1290L & $1 *$ & & & & & & \\
\hline $\mathrm{P} 480 \mathrm{~T}$ & 5 & & & $\mathrm{~T} 539 \mathrm{~A} / \mathbf{M 5 6 0 T} \quad(\mathrm{AD})$ & 1 & G1292D & 1 & & & & & & \\
\hline $\mathrm{P} 480 \mathrm{H}$ & 1 & & & & & V1293I & 3 & & & & & & \\
\hline T539A & 4 & & & & & G1306A & 1 & & & & & & \\
\hline M560T & 4 & & & & & G1306V & 2 & & & & & & \\
\hline E950K & $1 *$ & & & & & $\mathrm{~T} 1313 \mathrm{M}$ & 7 & & & & & & \\
\hline & & & & & & L1362P & 1 & & & & & & \\
\hline & & & & & & M1370V & 3 & & & & & & \\
\hline & & & & & & intron 21 & 1 & & & & & & \\
\hline & & & & & & R1448P & 1 & & & & & & \\
\hline & & & & & & $\mathrm{R} 1448 \mathrm{H}$ & 1 & & & & & & \\
\hline & & & & & & $\mathrm{R} 1448 \mathrm{C}$ & 4 & & & & & & \\
\hline & & & & & & $\mathrm{R} 1448 \mathrm{G}$ & 1 & & & & & & \\
\hline & & & & & & G1456E & 2 & & & & & & \\
\hline & & & & & & M1476T & 1 & & & & & & \\
\hline & & & & & & Q1633E & 1 & & & & & & \\
\hline & & & & & & E1702K & 1 & & & & & & \\
\hline
\end{tabular}

(nc) in the CLCN1 heterozygous column: non-confirmatory with autosomal dominant inheritance (sporadic or incomplete segregation study)

*: Variants in CLCN1 and SCN4A identified in the same proband. (AD) in the CLCN1 compound heterozygous column: variants identified in a family with autosomal dominant inheritance with the dominant variant in bold. 
Table 2. Patient characteristic and mutated gene

\begin{tabular}{llll}
\hline & HypoPP & MC & Nav-Myt \\
\hline Total, n & 11 & 18 & 12 \\
Age, median (IQR) & $31(23-56)$ & $38.5(25.5-45.5)$ & $36.5(22.25-51)$ \\
Gender, male, n (\%) & $9(81.1)$ & $13(72.2)$ & $9(75)$ \\
Mutation (n) & $C A C N A 1 S \quad(7)$ & $C L C N 1$ only (17) & $S C N 4 A$ PMC/SCM (9) \\
& $S C N 4 A \quad(4)$ & & $S C N 4 A$ HyperPP (3) \\
\hline
\end{tabular}


Table 3. Quality of life data with INQoL

\begin{tabular}{lcccc}
\hline INQoL \& subscale & HypoPP & MC & Nav-Myt & $P$ value \\
\hline Muscle weakness & $36.8(0-73.7)$ & $0(0-39.5)$ & $0(0-60.5)$ & 0.2927 \\
Pain & $0(0-47.4)$ & $0(0-22.4)$ & $7.89(0-28.9)$ & 0.7889 \\
Fatigue & $26.3(0-36.8)$ & $23.7(0-64.5)$ & $10.5(0-44.7)$ & 0.7445 \\
Myotonia & $0(0-36.8)$ & $42.1(19.7-80.3)$ & $42.1(28.9-60.5)$ & $0.0099 *$ \\
Blepharoptosia & $0(0)$ & $0(0)$ & $0(0-3.95)$ & 0.3122 \\
Diplopia & $0(0)$ & $0(0)$ & $0(0)$ & 0.1838 \\
Dysphagia & $0(0)$ & $0(0-13.2)$ & $0(0-13.2)$ & 0.4736 \\
Activities & $25.0(0-50)$ & $27.3(11.1-67.1)$ & $21.3(17.4-37.7)$ & 0.7982 \\
Independence & $16.7(0-38.9)$ & $5.56(0-17.4)$ & $8.33(0.694-18.7)$ & 0.8103 \\
Relationships & $13.9(0-31.5)$ & $7.41(0-45.8)$ & $16.2(1.85-20.8)$ & 0.9213 \\
Emotions & $27.8(5.56-63.9)$ & $29.2(7.64-75)$ & $34.7(11.1-69.4)$ & 0.9953 \\
Body image & $8.33(0-44.4)$ & $22.2(0-45.8)$ & $20.8(16.7-43.7)$ & 0.4589 \\
INQoL index & $26.7(0-63.9)$ & $24.4(5.83-56.4)$ & $33.1(18.7-44.4)$ & 0.9497 \\
\hline
\end{tabular}

INQoL subscale score comparisons among three groups of skeletal muscle channelopathy by Kruskal-Wallis test. The scores show the median and interquartile range in parentheses. It suggests that HOP group has less influence of myotonia than MC group and SCM group. 
Table 4. Correlation between INQoL index and INQoL section1 subscale

\begin{tabular}{|c|c|c|c|c|c|c|}
\hline \multirow[b]{2}{*}{$\begin{array}{l}\text { INQoL } \\
\text { subscale }\end{array}$} & \multicolumn{2}{|c|}{ НуроРР } & \multicolumn{2}{|c|}{ MC } & \multicolumn{2}{|c|}{ Nav-Myt } \\
\hline & $\begin{array}{c}R- \\
\text { Spearman }\end{array}$ & $P$ value & $\begin{array}{c}R- \\
\text { Spearman }\end{array}$ & $P$ value & $\begin{array}{c}\boldsymbol{R}- \\
\text { Spearman }\end{array}$ & $P$ value \\
\hline Muscle weakness & 0.8001 & 0.0031 & 0.3908 & 0.1089 & 0.6171 & 0.0325 \\
\hline Pain & 0.5219 & 0.0996 & 0.4206 & 0.0822 & 0.2716 & 0.3931 \\
\hline Fatigue & 0.9175 & $<.0001$ & 0.5690 & 0.0137 & 0.3571 & 0.2545 \\
\hline Myotonia & 0.2338 & 0.489 & 0.8085 & $<.0001$ & 0.1944 & 0.545 \\
\hline Blepharoptosia & 0.3137 & 0.3475 & -0.2572 & 0.3028 & 0.2804 & 0.3773 \\
\hline Diplopia & 0.3137 & 0.3475 & $\mathrm{n} / \mathrm{a}$ & $\mathrm{n} / \mathrm{a}$ & -0.1077 & 0.739 \\
\hline Dysphagia & 0.3770 & 0.2531 & 0.3204 & 0.1949 & 0.3092 & 0.3281 \\
\hline
\end{tabular}

Spearman correlation estimates between INQoL index calculated from INQoL section 2 subscales and INQoL section1 subscale. It suggests that INQoL index of HOP group correlates with muscle weakness and fatigue, MC group dose with fatigue and myotonia and SCM group dose with muscle weakness. Because no one has diplopia in MC group, correlation coefficient and $\mathrm{p}$ value were not calculated. 


\begin{tabular}{lcccc}
\hline Questionnaire and subscale & HypoPP & MC & Nav-Myt & $P$ value \\
\hline Physical functioning & $90.0(70-100)$ & $82.5(72.5-95.0)$ & $92.5(47.5-100)$ & 0.6263 \\
Physical role functioning & $100(68.8-100)$ & $90.6(54.7-100)$ & $84.4(31.3-93.8)$ & 0.3966 \\
Bodily pain & $100(74-100)$ & $73.0(59.5-100)$ & $79(32-100)$ & 0.4653 \\
General health perceptions & $62.0(55-72)$ & $64.5(46.5-83.3)$ & $58.5(21.3-75.8)$ & 0.3781 \\
Vitality & $58.1(43.8-87.5)$ & $56.3(25-68.8)$ & $56.3(14.1-78.1)$ & 0.5682 \\
Social functions & $100(62.5-100)$ & $100(59.4-100)$ & $87.5(25-100)$ & 0.5475 \\
Role emotions & $100(83.3-100)$ & $100(79.2-100)$ & $91.7(29.2-100)$ & 0.4522 \\
Mental health & $75.0(60-95)$ & $67.5(53.8-82.5)$ & $65(55-88.8)$ & 0.4126 \\
Mental component score & $52.7(44.9-65.6)$ & $48.8(41.8-56.3)$ & $45.5(36.6-56.5)$ & 0.3789 \\
Physical component score & $52.8(41.3-57.2)$ & $47.4(37.4-56.6)$ & $51.3(18.3-54.9)$ & 0.8406 \\
\hline
\end{tabular}

SF-36 subscale score comparisons among three groups of skeletal muscle channelopathy by Kruskal-Wallis test. The scores show the median and interquartile range in parentheses. 
Table 6. Comparison between the etiological data in this study and previous publications.

\begin{tabular}{|c|c|c|c|c|c|c|c|}
\hline & $\begin{array}{c}\text { Japan } \\
\text { (This study) }\end{array}$ & $\begin{array}{l}\text { England } \\
\text { (Ref 26) }\end{array}$ & $\begin{array}{c}\text { UK } \\
\text { (Ref 32) }\end{array}$ & $\begin{array}{c}\text { UK } \\
\text { (Ref 31) }\end{array}$ & $\begin{array}{c}\text { Netherlands } \\
\text { (Ref 25) }\end{array}$ & $\begin{array}{l}\text { Canada } \\
\text { (Ref 24) }\end{array}$ & $\begin{array}{c}\text { Italy } \\
\text { (Ref 27) }\end{array}$ \\
\hline NDM & $63 \%(66)$ & $71 \%(322)$ & & & $80 \%(188)$ & & (526) \\
\hline PP & $37 \%(39)$ & $29 \%(131)$ & & & $20 \%(48)$ & & (94) \\
\hline NDM- MC (CLCNI) & $45 \%(30)$ & $78 \%(252)$ & & & $57 \%(108)$ & $72 \%(36)$ & $73 \%$ \\
\hline MC-Dominant & $67 \%(20)$ & & & & 4 mutations & $25 \%(9)$ & \\
\hline MC-Recessive & $20 \%(6)$ & & & & majority & $75 \%(27)$ & \\
\hline NDM- SCM/PMC (SCN4A) & $55 \%(36)$ & $22 \%(70)$ & & & $43 \%(80)$ & $28 \%(14)$ & $27 \%$ \\
\hline HyperPP $(S C N 4 A)$ & (11) & (48) & & & (7) & & (34) \\
\hline hypoPP1 (CANCA1S) & $57 \%(16)$ & $80 \%(47)$ & $92 \%(11)$ & $77 \%(64)$ & $74 \%(26)$ & & \\
\hline hypoPР2 (SCN4A) & $43 \%(12)$ & $20 \%(12)$ & $8 \%(1)$ & $23 \%(19)$ & $26 \%(9)$ & & \\
\hline ATS & & (24) & & & (6) & & (15) \\
\hline
\end{tabular}




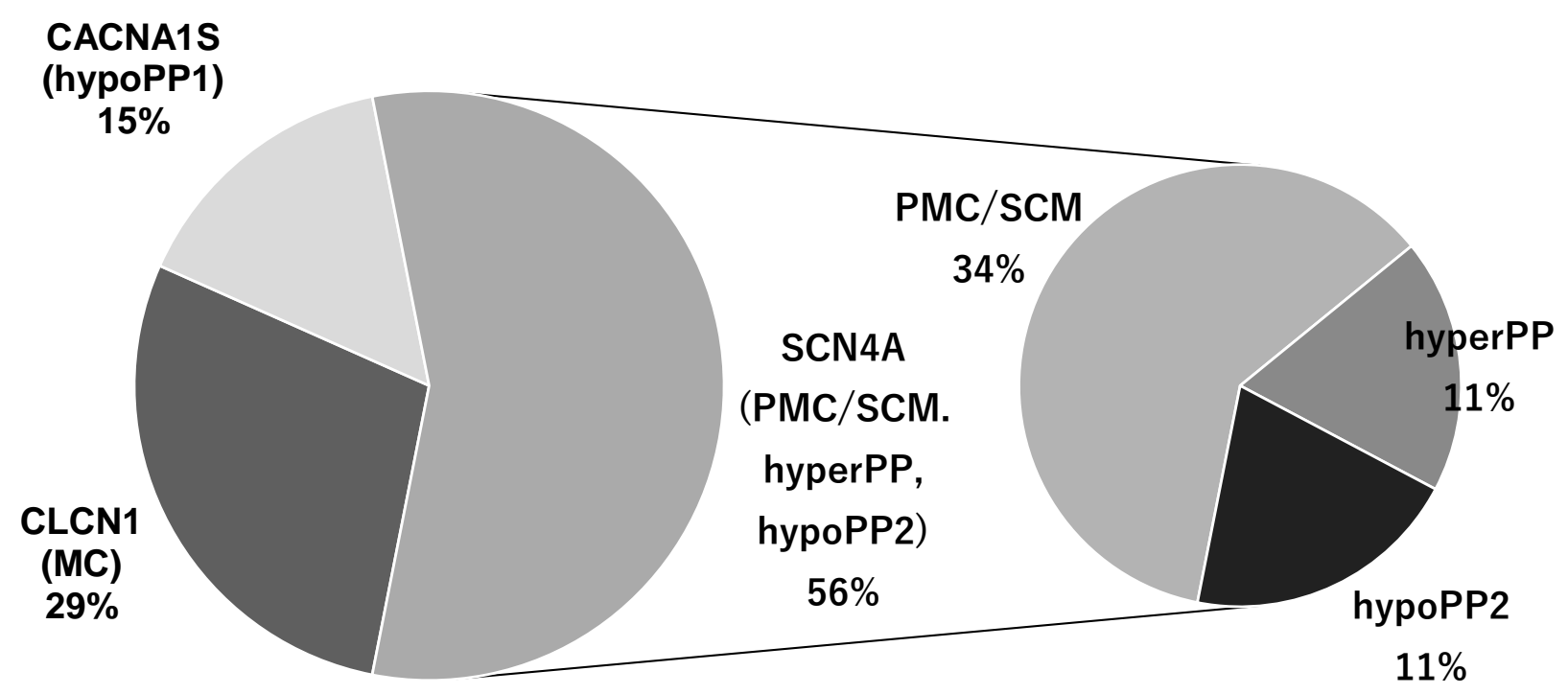

$\square$ MC $\square$ PMC/SCM $\square$ hyperPP $\square$ hypoPP2 $\square$ hypoPP1 
Supplemental figure 1. Family trees with heterozygous mutations in CLCN1 gene in Japan
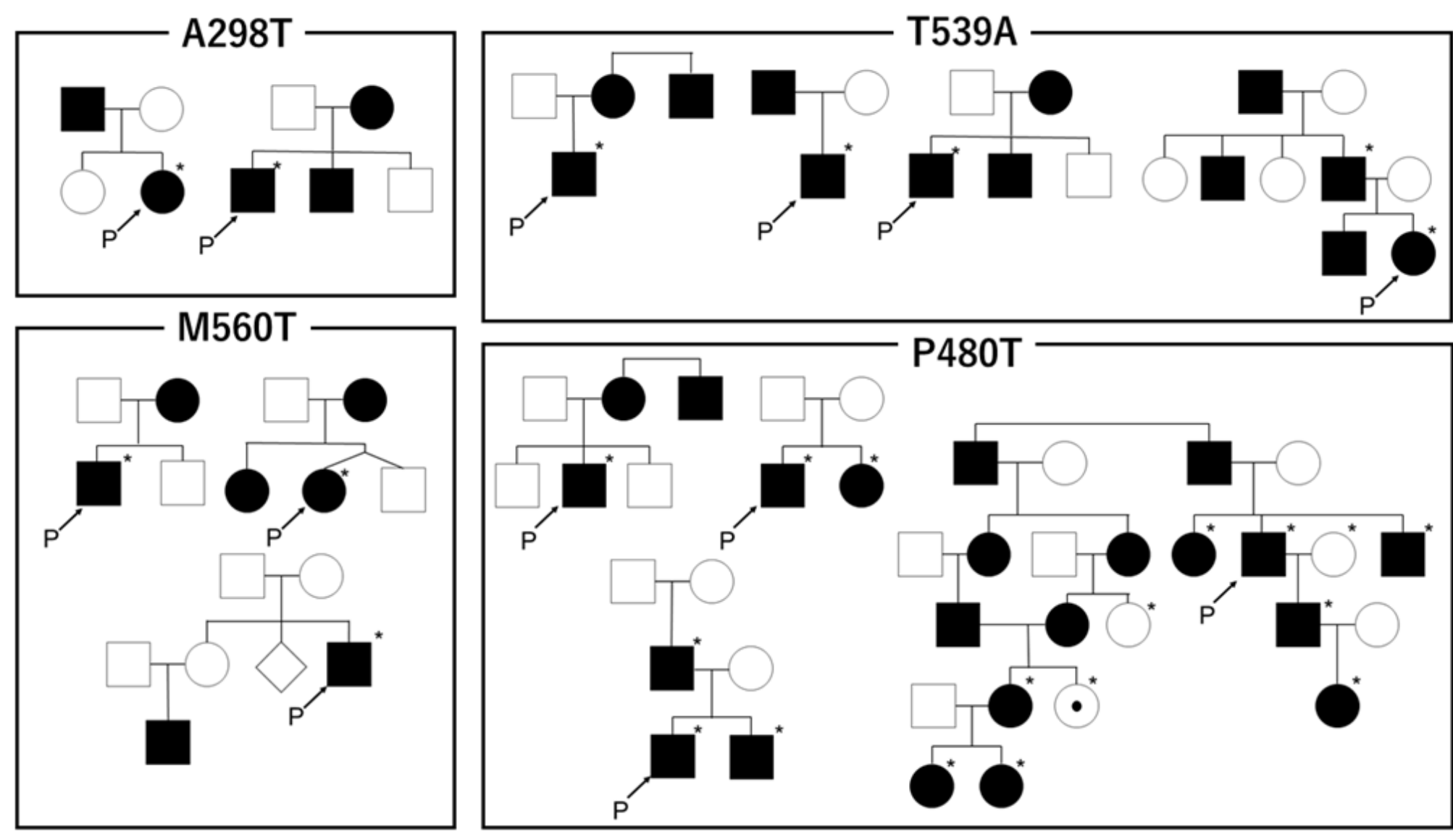

P480T

Representative family trees with heterozygous mutations in CLCN1 gene in Japan are shown. Filled marks indicate family members with clinical myotonic symptom based on medical interviews and/or evaluations. Probands in each family is highlighted in mark "P". Asterisks indicate individuals who were confirmed to have the mutation by genetic analysis.

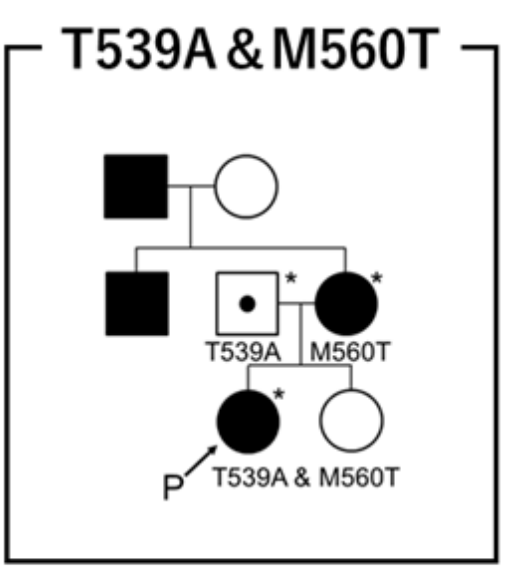

P480T \& R626X

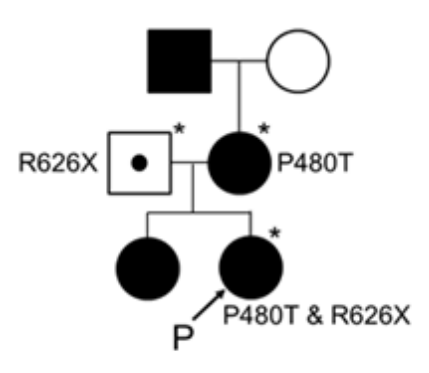


Supplemental Table 1. Allele frequency in a database and literature cases for major CLCN1 variants identified in this study.

\begin{tabular}{|c|c|c|c|c|c|c|}
\hline \multirow{2}{*}{ Variants } & \multicolumn{2}{|c|}{ Frequency in gnomAD } & \multicolumn{2}{|c|}{ Literature cases } & \multirow[b]{2}{*}{ ethnicity } & \multirow[b]{2}{*}{ Ref. } \\
\hline & all & East Asian & inheritance & zygosity & & \\
\hline \multirow{2}{*}{ A298T } & \multirow{2}{*}{$4 / 251390$} & \multirow{2}{*}{$3 / 18394$} & $\mathrm{AD}$ & hetero & Chinese & [1] \\
\hline & & & $\mathrm{AD}$ and $\mathrm{S}$ & $?$ & Korean & [2] \\
\hline $\mathrm{P} 480 \mathrm{~T}$ & None & - & $\mathrm{AD}$ & hetero & Japanese & [3] \\
\hline T539A & $1 / 251402$ & $0 / 18394$ & & - & & \\
\hline \multirow{4}{*}{ M560T } & \multirow{4}{*}{$1 / 251096$} & \multirow{4}{*}{ 0/18394 } & AR & compound hetero & Chinese & [4] \\
\hline & & & $\mathrm{AR}$ & hetero & Chinese & [4] \\
\hline & & & $\mathrm{AD}^{*}$ & compound hetero? & Chinese & [1] \\
\hline & & & $?$ & hetero & Korean & [5] \\
\hline
\end{tabular}

$\mathrm{S}$ : sporadic, $\mathrm{AD}$ : autosomal dominant, $\mathrm{AD}^{*}$ : autosomal dominant with reduced penetrance

[1] F. Gao, F.C. Ma, Z.F. Yuan, C.W. Yang, H.F. Li, Z.Z. Xia, Q.X. Shui, K.W. Jiang, Novel chloride channel gene mutations in two unrelated Chinese families with myotonia congenita., Neurol. India. 58 (2010) 743-6. doi:10.4103/0028-3886.72163.

[2] H.J. Chin, C.H. Kim, K. Ha, J.H. Shin, D.-S. Kim, I. So, Electrophysiological characteristics of R47W and A298T mutations in CLC-1 of myotonia congenita patients and evaluation of clinical features., Korean J. Physiol. Pharmacol. 21 (2017) 439-447.

doi:10.4196/kjpp.2017.21.4.439. 
[3] A. Tsujino, M. Kaibara, H. Hayashi, H. Eguchi, S. Nakayama, K. Sato, T. Fukuda, Y. Tateishi, S. Shirabe, K. Taniyama, A. Kawakami, A CLCN1 mutation in dominant myotonia congenita impairs the increment of chloride conductance during repetitive depolarization, Neurosci. Lett. 494 (2011) 155-160. doi:10.1016/j.neulet.2011.03.002.

[4] X.-L. Liu, X.-J. Huang, J.-Y. Shen, H.-Y. Zhou, X.-H. Luan, T. Wang, S.-D. Chen, Y. Wang, H.-D. Tang, L. Cao, Myotonia congenita: novel mutations in CLCN1 gene., Channels (Austin). 9 (2015) 292-8. doi:10.1080/19336950.2015.1075676.

[5] T.-S. Nam, H.-J. Jung, S.-Y. Choi, Y.-O. Kim, M.-K. Kim, K.-H. Cho, Clinical Characteristics and Analysis of CLCN1 in Patients with \&quot;EMG Disease\&quot;., J. Clin. Neurol. 8 (2012) 212-7. doi:10.3988/jcn.2012.8.3.212. 
Supplemental table 2. Comparison with Norm-based scoring in SF-36

It shows Norm-based scoring in SF-36. The scores show median and interquartile range in parentheses. The subscales are normalized by estimating the national average each subscale to 50 points and the standard deviation to 10 points.

\begin{tabular}{llll}
\hline SF-36 subscale & HypoPP & MC & Nav-Myt \\
\hline Physical functioning & $50.6(36.2-57.8)$ & $45.2(38.0-54.2)$ & $52.4(19.9-57.8)$ \\
Physical role functioning & $55.7(39.1-55.7)$ & $50.7(31.6-55.7)$ & $47.4(19.2-52.4)$ \\
Bodily pain & $61.7(50.1-61.7)$ & $49.7(43.6-61.7)$ & $52.3(31.4-61.7)$ \\
General health perceptions & $49.5(45.8-54.8)$ & $50.9(41.3-60.8)$ & $47.7(27.8-56.8)$ \\
Vitality & $47.6(40.2-62.7)$ & $46.6(30.6-53.0)$ & $46.6(24.9-57.9)$ \\
Social role functioning & $57.0(37.7-57.0)$ & $57.0(36.1-57.0)$ & $50.6(18.4-57.0)$ \\
Emotional role functioning & $56.1(47.8-56.1)$ & $56.1(45.7-56.1)$ & $51.9(20.7-56.1)$ \\
Mental health & $51.8(43.8-62.6)$ & $47.8(40.4-55.9)$ & $46.5(41.1-59.2)$ \\
Physical component score & $49.0(44.8-55.6)$ & $49.2(41.3-58.4)$ & $47.4(35.4-56.5)$ \\
Mental component score & $51.0(43.9-65.4)$ & $49.9(43.9-56.2)$ & $44.3(40.4-56.4)$ \\
Role-social component score & $49.5(36.1-55.4)$ & $48.1(35.2-56.5)$ & $49.4(20.6-56.9)$ \\
\hline
\end{tabular}

